\title{
Modeling effectiveness analysis of momentum and heat transfer in a fixed bed silo-dryer through computational fluid-dynamics
}

\author{
Felipe Leonardo Barcelos Mateus ${ }^{1}$ (B), Irineu Petri Júnior ${ }^{1}$ (B)
}

\author{
${ }^{1}$ Universidade Federal de Lavras/UFLA, Departamento de Engenharia/DEG, Lavras, MG, Brasil \\ "Corresponding author: felipe.lb.mateus@gmail.com; irineu.junior@ufla.br \\ Received in March 11, 2021 and approved in September 20, 2021
}

\begin{abstract}
The drying procedure is of great use in the industry, being capable of increasing the shelf life of many products, in addition to facilitate its transport and storage. The operation is essential for coffee beans to remove their excessive moisture content. The silo-drier has potential to ensure sensorial qualities through the intermittent drying, as well as with optimizations in the energy consumption. The objective of this work was to study the optimization of the numeric representation of a silo-dryer system, comparing the results of three different models available in the software FLUENT ${ }^{\circ}$ 19.2: Eulerian Multiphase Model for Granular flow with packed bed configuration disabled, the same model but with the packed bed configuration enabled, and the porous zone single-phase modelling. The convergence efficiency of these models was also analyzed through the results of the numeric residue, and the influence of the relaxation factor in the simulation results. The behavior of solid volumetric fraction, air velocity and bed temperature were qualitatively examined with hot air at the inlet. It was found that the momentum relaxation factor influenced the results. Moderately lower factors allowed for faster convergence without significant particle behavior change. Monitoring the mass residue, the enabled packed bed modelling presented lower residuals, while the porous zone modelling presented higher residuals. The packed bed and porous zone modelling reported lower air velocities in comparison to the disabled packed bed modelling, and presented satisfactory heat transfer through the temperature profiles.
\end{abstract}

Key words: Cfd; Coffee; Drying; Eulerian; Multiphase.

\section{INTRODUCTION}

Coffee is a dominant product in the Brazilian market, where coffee seeds are exported at great quantities. In 2019, the total coffee export was of 2 million tons, in an average monthly value of 400 million dollars according to Coffee Statistical Report (2019).

The quality of the coffee, its scent, coloration and taste, depends on its processing from harvest to the drink production. One of the crucial factors to guarantee the quality of the coffee is the drying procedure. Seed drying is necessary to prevent compromising effects to the stored product caused by the moisture content. After the harvest, the average moisture content of the coffee is $55 \%$ w.b., which needs to be reduced to approximately 11\% w.b. (Ghosh, 2014).

In drying, it is essential to control air and seed temperature, air velocity inside the equipment, and particle movement. For seed storage, the granular material temperature, as well as its moisture content are decisive to the activity of biotic components, which can seriously compromise the product (Resende et al., 2008).

Together with the control of the variables, it is necessary to investigate seed properties as to choose the better suited equipment for drying, in order to guarantee the quality of the product and maintain its physical properties. Common drying equipment are the fluidized bed and spouted bed, as they provide high velocities to intensify the mass and heat transfer. However, according to Soponronarit et al. (2001), for seeds with moisture content lower than 33\% d.b., mechanical stress is not recommended since it can compromise the physical structure of the seeds.

Consequently, an alternative is the use of fixed bed dryers, given the seeds are immobile inside the equipment, preventing major mechanical stress to the seeds. By combining both drying and storage procedures, the silo-dryer is capable of controlling the storage temperature simultaneously to the drying operation, potentially reducing damages to the material and operation costs (Coradi; Lemes, 2019). Palacin et al. (2009) concluded experimentally that the use of silo-dryers can reduce the energy consumption for coffee cherry seeds drying.

Another applicability of the silo-dryer, as proposed by Difendi, Paraíso and Jorge (2018), is the implementation of the intermittent drying in contrast to the usual procedure of continuous drying. The material, arranged inside its storage unity, can be dried in non-continuous periods within a long period of time. This allows for a better control of the procedure by allowing periodical modifications to the drying air properties. This way, the partitioning of the drying time shows potential to the optimization of the operation in terms of the product quality and the power consumption (Borém et al., 2014).

To better comprehend the drying process in a silo-dryer, the computational fluid-dynamics (CFD) tool can be useful as it allows for a differential analysis of the variables along the entirety of the equipment and along the operation. CFD is regarded as an integral part of engineering design and analysis 
for its predicting capabilities, and it has been used extensively in the food industry for operations such as sterilisation, mixing, refrigeration, and drying (Xia; Sun, 2002).

For the silo-drying modelling, it is possible to utilize the Eulerian Multiphase Model for Granular (EMMG) flow considering the system is constituted by air and particles. The EMMG considers both the phases to be interpenetrating fluids, which adds the concept of volumetric fraction to the system. Another possible modelling is the porous zone model, which considers the particle to be a static porous zone by which the drying air interpenetrates. In this model, the solid phase is not represented through discrete volumes, but through the addition of momentum drops for the fluid phase (Wang et al., 2010). In this case, the multiphase modelling is not necessary, as the continuity, momentum and energy equations are only applied to the drying air.

Amaral et al. (2018) studied the experimental coffee drying in a fixed bed dryer and the CFD application for coffee drying through the simulation of the moisture content in the system. The energy and momentum transfer was not studied. By simulating a symmetrical fraction of a coffee bean, Afonso et al. (2018) were able to fit the experimental data to the twoterm exponential mathematical model.

Stoppe, Neto, and Santos (2020) studied experimentally and numercally the fixed bed drying in a solar dryer. The simulations, however, only encompassed the mass and momentum analysis, without a numeric representation for the heat transfer. The CFD application in a fixed bed silo-dryer was studied by Coradi and Lemes (2018) through a bidimensional mesh, applied to soy seeds drying. The simulation was made considering the momentum and heat transfer of the system. They noted a more efficient energy consumption for the equipment, and described it as a viable and economic tool. However, the use of a bidimensional mesh might not be optimal, since it simplifies the system by disregarding some forces that are only applicable in tridimensional meshes, thus not representing the system as accurately as possible (Versteeg and Malalasekera).

Szafran and Kmiec (2004) studied the momentum, heat, and mass transfer in a spouted bed dryer, and obtained good results for the mass transfer, but the heat transfer was underpredicted. Other authors have also studied the use of CFD in fluidized bed dryers, such as Wang et al. (2008) and Hamzehei (2011), and obtained results converging to the experimental values. The study of fixed bed dryers, however, is still in development in the literature.

This work was based on the experimental and numerical study on soy seed drying by Coradi and Lemes (2019). By using the same initial and boundary conditions, as well as representing the equipment as described in their study, this work aims at formulating a similar numeric methodology applicable to coffee beans. For that, different models and their numeric efficiency are investigated so that the coffee bean simulation can be properly optimized.

Thus, the objective of this work was to predict, through CFD, the momentum and heat transfer in a cylindric silo-dryer, in order to evaluate the efficiency of the multiphase and porous zone models, as well as changes to the momentum relaxation factor. This was made in order to optimize the fixed bed modelling, by approximating the simulation behavior to the real system and reducing computational costs through the reduction of the residual of the simulation. With that, it is possible to evolve the numerical method to make predictions for the moisture content mass transfer, establishing a numerical method of low computational cost with good convergence for seed drying. Thus, the simulation can be an alternative or complementary tool to experimental tests, as it has low material and operation expenses, and through it, provide an important contribution to coffee drying.

\section{MATERIAL AND METHODS}

The geometry of the equipment was created in the software GAMBIT ${ }^{\circledR}$, determined by the silo-dryer constructed by Coradi and Lemes (2019), a cylindrical dryer with $1.5 \mathrm{~m}$ of height and $0.2 \mathrm{~m}$ of diameter. The GAMBIT ${ }^{\circledR}$ software was chosen for allowing a precise manual construction of the mesh, which permitted better cell regularity.

The mesh construction is of great importance to guarantee the proper convergence for the equipment. In order to assure a good mesh and representative results it is necessary to formulate a minimum number of cells and a regular shape for the cells (Versteeg; Malalasekera, 2007). The mesh was created from the cylindrical shape of the equipment with exclusively hexahedral cells, which have better geometrical regularity.

Three meshes were constructed, constituted by roughly 100,000, 200,000 and 300,000 cells, in order to ensure mesh independence for the final mesh. The parameters used in the mesh independence test consisted of the bean volumetric fraction along the simulation for all three meshes in an interval of 40 seconds of simulation. The final mesh was constituted by 210,888 cells, and can be observed in Figure 1.

It is important to note that, for the scope of this work, the properties of the particle are not of utmost importance for the convergence analysis. In order to be applicable to various uses of the silo-dryer, the analysis proposed here strives to achieve qualitative results that are not strictly dependent to the mentioned values.

The initial and boundary conditions for the simulation were configured in the software Ansys FLUENT ${ }^{\circledR}$ 19.2. These, aswell as other properties and submodels of the simulation are presented in Table 1. 


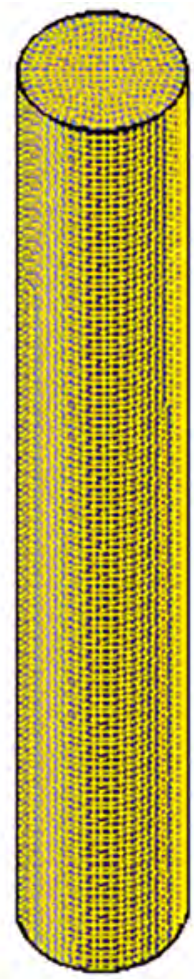

Figure 1 - Computational mesh of the silo-dryer.

The convergence criteria were of 10-6 for the energy balance residuals and 10-3 for the other residuals. The time step size was of 10-6 at the start of the simulation to guarantee a stable residual behavior, increasing to a maximum value of 10-4 at later simulation times.

\subsection{Mathematical modelling}

In this work three types of modelling were studied in order to predict the flow inside the silo-dryer, two regarding the EMMG, widely used in the literature (Chiesa et al., 2005; Ismail et al. 2016; Vicente et al., 2003) and one regarding the porous zone modelling, which uses the Darcy equations.

The modelling for the heat exchange was the same for all three models. The heat exchange coefficient between the phases can be modelled through the correlation expressed by Equation 1 (Mujumdar, 2006), utilized for perpendicular air flux interpenetrating a granular solid, where Gy $\left[\mathrm{kg} \cdot \mathrm{m}^{-2} \cdot \mathrm{s}^{-1}\right]$ is the air mass flux and $\mathrm{h}_{\mathrm{y}}\left[\mathrm{W} \cdot \mathrm{m}^{-2} \cdot \mathrm{s}^{-1}\right]$ the heat exchange coefficient. The heat transfer coefficient used was of 193.86 W.m-2 $\mathrm{K}^{-1}$. This value is used by the simulation to obtain the volumetric rate of energy transfer between the phases $\left(Q_{p q}\right)$ as shown in Equation 2, in which $\mathrm{A}_{\mathrm{i}}$ is the interfacial area between the phases and $\mathrm{T}_{\mathrm{p}}$ and $\mathrm{T}_{\mathrm{q}}$ are the temperatures of each phase. These temperatures vary over time because of the heat exchange between the phases, and this variation is modelled by the conservation of energy in Equation 3, where $\mathrm{c}_{\mathrm{p}}$ is the specific heat [ J.kg$\left.{ }^{1} \cdot \mathrm{K}^{-1}\right], \mathrm{S}_{\mathrm{h}}$ the energy generation or consumption in the system [W] and the time [s].

$h_{y}=24.2 \cdot G_{y}^{0.37}$

$Q_{p q}=h_{y} A_{i}\left(T_{p}-T_{q}\right)$

$\rho c_{p} \frac{\partial T}{\partial t}=-\nabla\left(Q_{p q}\right) \pm S_{h}$

The energy equation, as well as the continuity and momentum equations are the fundamental equations of fluid mechanics, and all three are used and solved by the solver, in this case, FLUENT ${ }^{\circledR}$, by the finite volumes method using the properties and boundary conditions previously presented in Table 1. The deduction of these equations and turbulence model can be described as following.

\subsubsection{Continuity Equation}

The continuity equation is derived from the conservation of mass. Through a control volume containing a fluid, the mass conservation can be described by Equation 4 .

$\frac{\partial \rho}{\partial t}+\nabla(\rho \vec{V})= \pm S_{m}$

Where: $\rho$ is the fluid density; $\mathrm{V}$ is the velocity vector; $\mathrm{t}$ is time; $\mathrm{S}_{\mathrm{m}}$ is the mass generation or consumption rate.

By opening the gradient of the equation, the Equation 5 can be obtained. Considering no generation or mass consumption, the simplified version can be obtained, as shown in Equation 6.

$\frac{\partial \rho}{\partial t}+\left(\frac{\partial \rho u}{\partial x}+\frac{\partial \rho v}{\partial y}+\frac{\partial \rho w}{\partial z}\right)= \pm S_{m}$

$\frac{\partial \rho}{\partial t}+\left(\frac{\partial \rho u}{\partial x}+\frac{\partial \rho v}{\partial y}+\frac{\partial \rho w}{\partial z}\right)=0$

Where $\mathrm{u}, \mathrm{v}$ and $\mathrm{w}$ are the velocity vector components for the $\mathrm{x}$, $\mathrm{y}$ and $\mathrm{z}$ axis respectively.

\subsubsection{Momentum Theorem}

The momentum theorem derives from the second law of Newton, which states that the liquid force applied to a system defines the momentum intensity and direction over time. The global balance of momentum can be generally represented by Equation 7.

$\sum F=\iint_{s . c .} v \rho(v \cdot n) d A+\frac{\partial}{\partial t} \iiint_{v . c .} \rho v d V$ 
Table 1 - Initial and boundary conditions, properties, and submodels used in the simulation.

\begin{tabular}{|c|c|c|}
\hline Boundary Conditions & & Reference \\
\hline Pressure at the outlet & $101325 \mathrm{~Pa}$ & \\
\hline Air temperature at outlet & $90^{\circ} \mathrm{C}$ & (Coradi et al., 2018) \\
\hline Air temperature at inlet & $90^{\circ} \mathrm{C}$ & (Coradi et al., 2018) \\
\hline Air interstitial velocity at inlet & $0.35 \mathrm{~m} . \mathrm{s}^{-1}$ & (Coradi et al., 2018) \\
\hline Shear condition at walls & No slip & \\
\hline Initial Conditions & & Reference \\
\hline Bed height & $0.75 \mathrm{~m}$ & \\
\hline Particle velocity & $0 \mathrm{~m} . \mathrm{s}^{-1}$ & \\
\hline Initial bed Temperature & $25^{\circ} \mathrm{C}$ & (Coradi et al., 2018) \\
\hline Particle volumetric fraction & 0.5236 & \\
\hline Particle Properties & & Reference \\
\hline Density & $791.2 \mathrm{~kg} \cdot \mathrm{m}^{-3}$ & (Welty; Rorrer; Foster, 2014) \\
\hline Viscosity & $1.789410^{-5} \mathrm{~kg} \cdot \mathrm{m}^{-1} \cdot \mathrm{s}^{-1}$ & \\
\hline Diameter & $0.01 \mathrm{~m}$ & (Amaral et al., 2018 \\
\hline Specific heat & $1856 \mathrm{~J} \mathrm{~kg}^{-1} \cdot \mathrm{K}^{-1}$ & Azadbakht et al., 2013; Borém et al., 2002 \\
\hline Termal conductivity & $0.038 \mathrm{~W} \cdot \mathrm{m}^{-1} \cdot \mathrm{K}^{-1}$ & Azadbakht et al., 2013; Borém et al., 2002) \\
\hline Air Properties & & Reference \\
\hline Density & $1.225 \mathrm{~kg} \cdot \mathrm{m}^{-3}$ & \\
\hline Viscosity & $1.789410^{-5} \mathrm{~kg} \cdot \mathrm{m}^{-1} \cdot \mathrm{s}^{-1}$ & \\
\hline Specific heat & $1006.43 \mathrm{~J} \mathrm{~kg}^{-1} \cdot \mathrm{K}^{-1}$ & \\
\hline Thermal conductivity & $0.0242 \mathrm{~W} \cdot \mathrm{m}^{-1} \cdot \mathrm{K}^{-1}$ & \\
\hline Multiphase Eulerian Granular Model property & Submodel & \\
\hline Granular viscosity & Syamlal-obrien & \\
\hline Granular bulk viscosity & Lun-et-al & \\
\hline Solids pressure & Lun-et-al & \\
\hline Granular temperature & Algebraic & \\
\hline Radial distribution & Lun-et-al & \\
\hline Elasticity modulus & Derived & \\
\hline Relaxation factor & & Reference \\
\hline Pressure & 0.3 & (Costa; Peres; Petri, 2021) \\
\hline Energy & 0.1 & (Costa; Peres; Petri, 2021) \\
\hline Momentum & $0.1,0.01$ and 0.001 & (Costa; Peres; Petri, 2021) \\
\hline
\end{tabular}

Where: $\mathrm{F}$ is force; $\vec{V}$ is the velocity vector; $\mathrm{n}$ is the normal vector; $\mathrm{A}$ is the area; $\mathrm{V}$ is the volume.

The resulting forces $\left(\sum \mathrm{F}\right)$ can be divided into the gravitational and superficial (pressure and viscous tensions) forces, these being the forces that influence the behavior of the fluid more significantly. Thus, by differentiating Equation 7, Equation 8 is obtained.

$\rho \frac{\partial \vec{V}}{\partial t}+\rho \vec{V} \cdot \nabla \vec{V}=\rho \vec{g}-\nabla P+\nabla \cdot \tau_{i j}+\vec{F}$
Where: $\mathrm{g}$ is the gravitational force; $\mathrm{P}$ is the pressure force; $F$ are the multiphase interaction forces; $\tau_{\mathrm{ij}}$ the viscous forces.

The viscous forces are modelled through the definitions for newtonian and incompressible fluids of Navier-Stokes, and by applying this consideration, Equation 9 is obtained. In this, the viscous forces are considered as proportional to a constant that represent the deforming rate of the fluid, the viscosity $(\mu)$.

$\rho \frac{D \vec{V}}{D t}=\rho \vec{g}-\nabla P+\mu \nabla^{2} \vec{V}+\vec{F}$ 


\subsubsection{Energy Equation}

The energy equation comes from the first law of thermodynamics, which sets the energy conservation law. It is also treated as a balance through a control volume, as described by Equation 10. Considering the normal tension effects are proportional to the effects of pressure and viscous effects, and by substituting the shaft work $\left(\mathrm{W}_{\mathrm{s}}\right)$, flow and shear stress $\left(\mathrm{W}_{\mu}\right)$ in the equation, Equation 11 is obtained.

$$
\begin{aligned}
& \frac{\delta Q}{\partial t}-\frac{\delta W}{d t}=\iint_{s . c .} e \rho(v \cdot n) d A+\frac{\partial}{\partial t} \iiint_{v . c .} e \rho d V \\
& \frac{\delta Q}{\partial t}-\frac{\delta W_{s}}{d t}=\iint_{\text {s.c. }}\left(e+\frac{P}{\rho}\right) \rho(v \cdot n) d A+\frac{\partial}{\partial t} \iiint_{v . c .} e \rho d V+\frac{\partial W_{\mu}}{\partial t}
\end{aligned}
$$

Where: $\mathrm{Q}$ is the transferred heat; $\mathrm{W}$ is the work; e is the energy per mass;

The energy per mass can be substituted by the internal, kinetic and potential energies. In this case, adopting a null shaft work, insignificant macroscopic movement, homogeneous media and constant physical properties, the energy can be defined through the enthalpy, pressure and volume of the system. With these considerations and also Fourier's law, Equation 13 can be simplified into equation 12 .

$\rho c_{v} \frac{\partial T}{\partial t}=\nabla \cdot(k \nabla T) \pm S_{h}$

Where: $\mathrm{c}_{\mathrm{v}}$ is the volumetric specific heat; $\mathrm{k}$ is the thermal conductivity; $\mathrm{S}_{\mathrm{h}}$ is the energy generation or consumption.

\subsubsection{Turbulence model}

The turbulence model used was the Reynolds Average

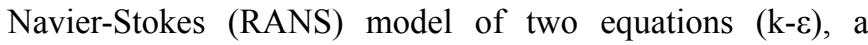
semi-emphirical model that solves the transport equation of the turbulent kinetics (Equation 13) and dissipation forces (Equation 14) separately.

$$
\begin{aligned}
& \frac{\partial(\rho k)}{\partial t}+\frac{\partial(\rho k u)}{\partial x_{1}}=\frac{\partial}{\partial x_{2}}\left[\left(\mu+\frac{\mu_{t}}{\sigma_{k}}\right) \frac{\partial k}{\partial x_{2}}\right]+G_{k}+G_{b}-\rho \varepsilon-Y_{M}+S_{k} \\
& \frac{\partial(\rho \varepsilon)}{\partial t}+\frac{\partial(\rho \varepsilon u)}{\partial x_{1}}=\frac{\partial}{\partial x_{2}}\left[\left(\mu+\frac{\mu_{t}}{\sigma_{\varepsilon}}\right) \frac{\partial \varepsilon}{\partial x_{2}}\right]+C_{1 \varepsilon} \frac{\varepsilon}{k}\left(G_{k}+C_{2 \varepsilon} G_{b}\right)-C_{2 \varepsilon} \rho \frac{\varepsilon^{2}}{k}+S_{\varepsilon}
\end{aligned}
$$

Where: $G_{k}$ is the kinetic energy generation through average velocity gradientes; $G_{b}$ is the kinetic energy generation through flotability; $\mathrm{Y}_{\mathrm{M}}$ is the floating dilatation contribution; $\boldsymbol{\alpha}_{\mathrm{k}}$ and $\boldsymbol{\alpha}_{\varepsilon}$ are the turbulent Prandtl numbers for $k$ and $\varepsilon ; S_{k}$ and $S_{\varepsilon}$ are terms defined by the user; $\mu_{t}$ is the turbulent viscosity; $\mathrm{C}_{1 \varepsilon}, \mathrm{C}_{2 \varepsilon}$ and $\mathrm{C}_{3 \varepsilon}$ are constants of the model.
Thus, the Multiphase Models can be used to consider phase calculations in the conservation equations. The first model, using EMMG, referred in this work as Model I, did not use the packed bed configuration, but disregarded the drag modelling for fluid-solid interactions. This was done as to try to better represent a fixed bed regime, that is, the regime where particles stay static in the bed. By disabling the drag, it helps to prevent particle movement inside the equipment just like the experimental operation. The second EMMG model, referred as Model II, used the packed bed configuration available in the software FLUENT ${ }^{\circledast}$ combined with fixed velocities of zero for the solid phase. In this model, the drag modelling is irrelevant. Both models use same continuity equations.

It is important to note that Model II still uses Equation 17 for the conservation of mass calculations, but freezes the interstitial velocity for the solid phase in a value of zero. For the momentum, the Navier-Stokes equations were used, as presented previously.

For Model III, the porous zone modelling was used, which uses the Darcy equations to predict the fluid flow through the porous media. This type of approach dismisses a multiphase modelling, since the cell conditions configured as a porous zone is treated as the solid phase, and only a fluid phase is defined. The continuity equation for the porous zone modelling treats $\mathrm{S}_{\mathrm{q}}$ as a function of the volumetric fraction of solid, viscosity, density, and the velocity of the fluid phase in all axes $\left(\mathrm{v}_{\mathrm{i}}\right)$. For this work, the porous zone was considered to be homogeneous, and so the $\mathrm{S}_{\mathrm{q}}$ for the solid phase is defined as $\mathrm{S}_{\mathrm{i}}$ (source term for the $\mathrm{i}$ axis), as shown in Equation 15. $\mathrm{C}_{2}$ is the inertial resistance factor, defined as $1161 \mathrm{~m}^{-1}$ according to Equation 16 (ANSYS, 2018).

$$
\begin{aligned}
& S_{i}=\left(\frac{\mu}{\alpha} v_{i}+C_{2} \frac{1}{2}|v| v_{i}\right) \\
& C_{2}=\frac{3.5(1-\varepsilon)}{D_{p} \varepsilon^{3}}
\end{aligned}
$$

The Reynolds Average Navier-Stokes (RANS) model of two equations $(\mathrm{k}-\varepsilon)$ was used. The RANS modelling approach to turbulence simulates the turbulent behaviour through the addition of a fluctuating value for properties such as velocity, temperature and pressure (Versteeg; Malalasekera, 2007).

The relaxation factor is a parameter used in computational simulations to dampen the calculated values at each numerical iteration in order to maintain stability and avoid divergences. As it directly influences the calculation of the simulation, its analysis is of great importance.

Model I has a higher computational effort for the numerical calculations of the momentum due to the particle movement. Even with the drag model disabled, the particles are still slightly moved by the air, which results in a higher 
computational load compared to the other models. Changes in the momentum relaxation factor could assist in reducing this load by adding inertia to the solid phase allowing for a faster convergence of the packed bed.

Thus, the effect of three different relaxation factors were made for the Model I, in order to study the efficiency of the change at stabilizing the numerical calculation. For the Models II and III the relaxation factor was defined as 0.1, recommended for transient state simulations (Santos, 2010).

The mass residual, which measures the convergence efficiency and the stability of the simulation, was monitored throughout the first 4 seconds of simulation for all three models. Subsequently, the simulations continued to be calculated until a simulation time of 42 seconds, where the volumetric fraction, particle temperature and air velocity profiles were obtained.

The computational cost of a simulation depends on the complexity of the models used and the total simulation time for transient simulations. The criteria used by the solver to consider a time step to have converged or not is the residual. Thus, its monitoring is fundamental, and as lower residuals implies faster converging time steps, it also implies an overall lower computational cost.

The results with different relaxation factors for Model I were compared, analyzing its efficiency at assisting the convergence of the problem. This was accompannied by the analysis of the air velocity and volumetric fraction profiles, in order to guarantee the converged state was not altered significally. Then, the three models were compared. Model I configured with a relaxation factor of 0.01 was compared to the results for Models II and III.

The volumetric fraction profiles for the EMMG are useful to observe the particle position inside the equipment. The volumetric fraction concept is not applied to Model III, as only air is simulated as a volume in the system and the multiphase modelling is not applicable.

\section{RESULTS}

The effects of the relaxation factor and numerical model changes were analyzed in two different topics. Firstly, the residual for three different momentum relaxation factors for Model I were examined in order to verify its influence on the convergence. Subsequently, the volumetric fraction and air velocity profiles were also compared for the three factors. The temperature profiles were not compared, as their behavior was equivalent. The second topic addressed the comparison between the three models using the residual and profile analysis for volumetric fraction, air velocity and particle temperature.

The three different meshes were used in identically configured simulations, and the particle position for the time periods of $1,10,20,30$ and $40 \mathrm{~s}$ was monitored through volumetric fraction profiles. The simulations used Model I with a relaxation factor of 0,1 , theoretically representing the least stable condition for particle movement in this work. The results obtained are presented in Figure 2.

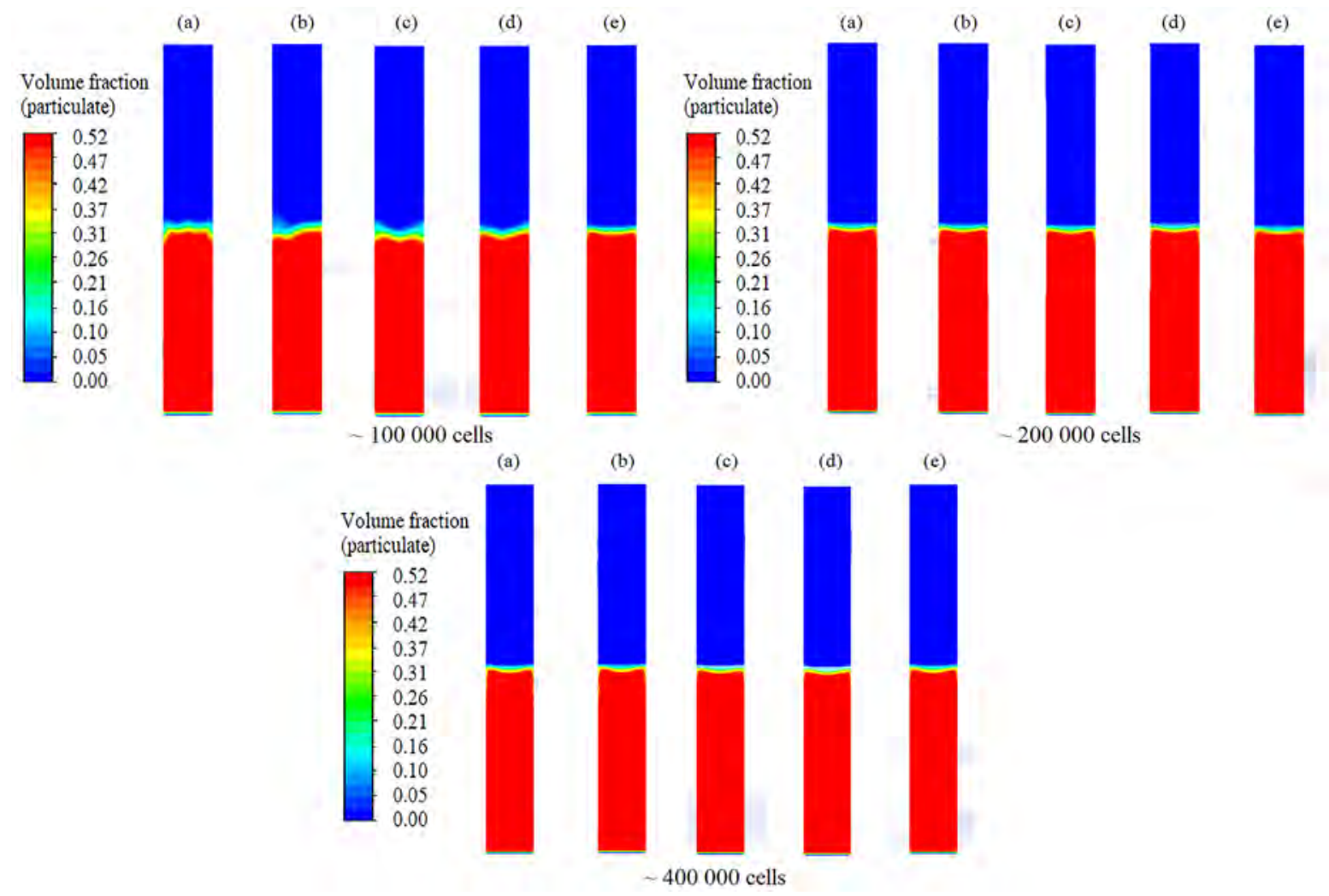

Figure 2 - Volumetric fraction profiles for (a) $1 \mathrm{~s}$, (b) $10 \mathrm{~s}$, (c) $20 \mathrm{~s}$, (d) $30 \mathrm{~s}$, (e) $40 \mathrm{~s}$. 
Three simulations of 4 seconds each using Model I were configured with different relaxation factors. The residual data for each simulation were monitored and compared. The obtained residuals for each relaxation factor can be observed in Figure 3.

The volumetric fractions obtained for each relaxation factor using Model I can be observed in Figure 4.

The air velocity profiles for Model I with different relaxation factors is presented in Figure 5. The black line represents the particle height inside the equipment.

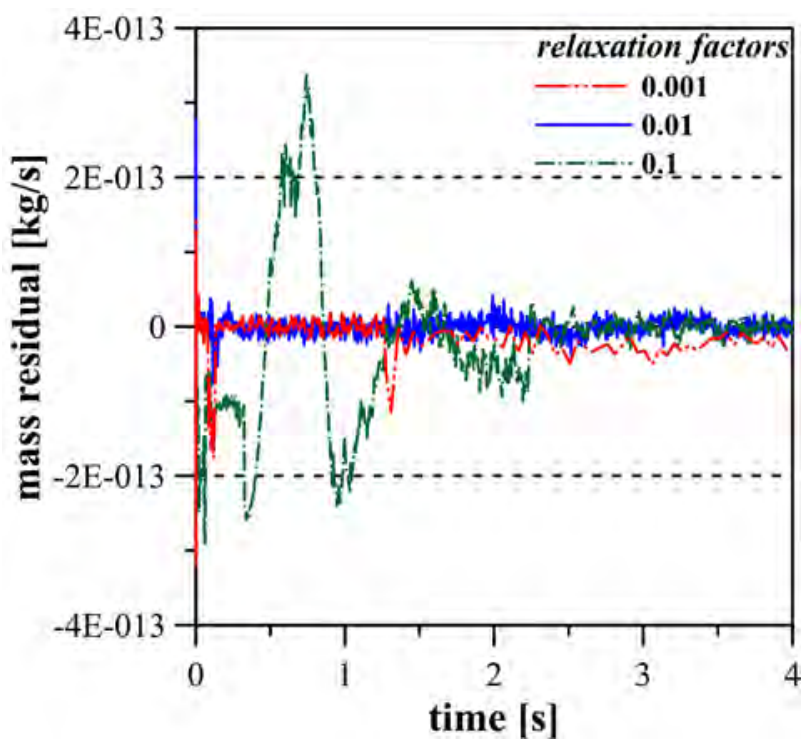

Figure 3 - Continuity residual for different relaxation factors using Model I. (a)

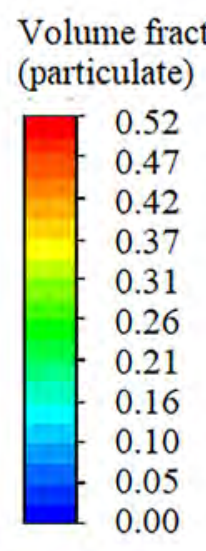

(b)

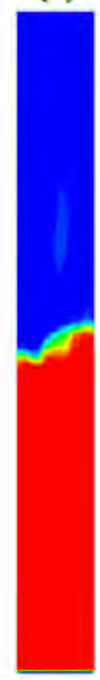

(c)

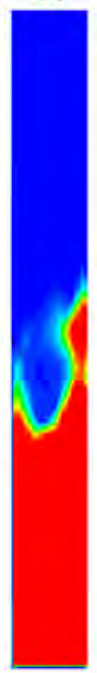

Figure 4 - Volumetric fraction profiles of the solid for Model I using relaxation factors of 0.1 (a), 0.01 (b) and 0.001 (c)

Two other simulations were calculated, each configured with different numerical models: Model II and Model III, both with a relaxation factor of 0.1 . The mass residual for both the simulations were compared to the mass residual obtained for Model I with a relaxation factor of 0.01 . This way, Model I is represented in the comparison at its best residual performance, since as shown in Section 3.1, the relaxation factor of 0.01 presented better residuals without significantly changing the simulation behavior. The residual for all three simulation in a period of 4 seconds of simulation is presented in Figure 6. (a)

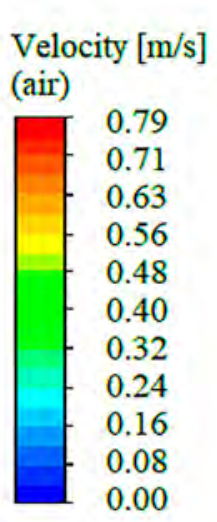

(b)

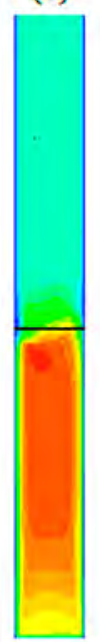

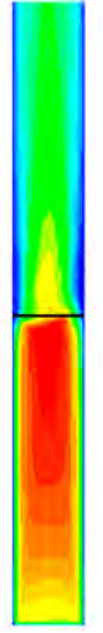

(c)

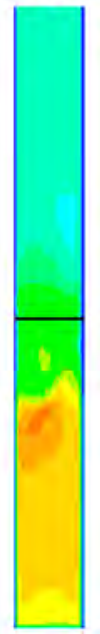

Figure 5 - Air velocity profiles for Model I using relaxation factors of $0.1(a), 0.01(b), 0.001$ (c)

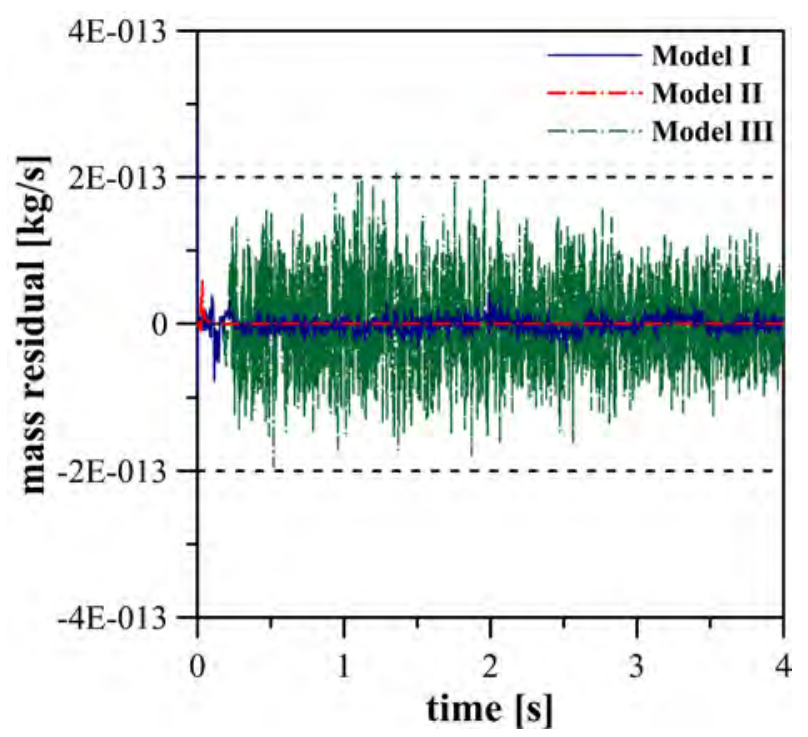

Figure 6 - Continuity residuals for Model I, II and III.

The volumetric fraction profiles for Model I and II is represented in Figure 7. The volumetric fraction profile for Model III was not presented, since, as explained in the numerical methodology, the volumetric fraction concept cannot be applied to the porous zone modelling. 
(a)

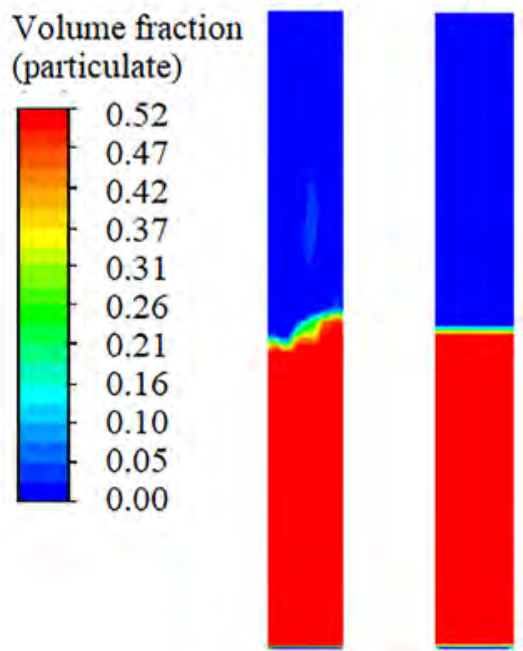

Figure 7 - Volumetric fraction profiles for Model I (a) and Model II (b).

The solid temperature profiles (in ${ }^{\circ} \mathrm{C}$ ) for all three models is presented in Figure 8. The velocity profiles (in $\mathrm{m} / \mathrm{s}$ ) for all three models are presented in Figure 9. (a)

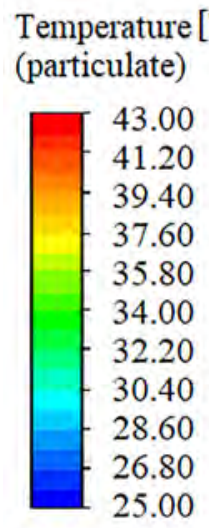

(b)

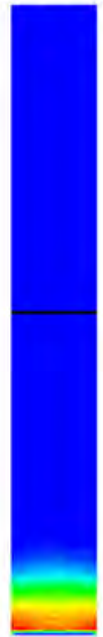

(c)

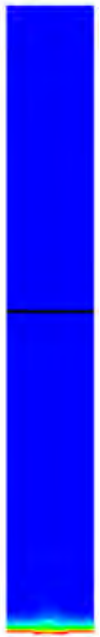

Figure 8 - Temperature profiles for Model I (a), Model II (b), and Model III (c).

\section{DISCUSSION}

\subsection{Mesh independence test}

The profiles showed in Figure 2 were similar for all meshes, with small variations to the particle behavior for the 100,000 cells mesh, in which the particle showed some movement close to the particle height. However, after approximately 40 seconds of simulation, the particles seemed to stabilize in a fixed bed behavior. Even so, considering some simulations used data from the first four seconds of simulation, the mesh of 200,000 cells was chosen as final mesh, since Figure 2 indicates the steady state behavior of the particles early on the simulation for the utilized mesh.

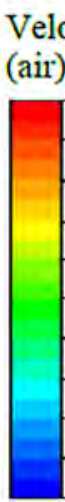

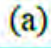

(a)

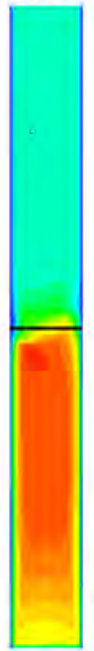

(b)

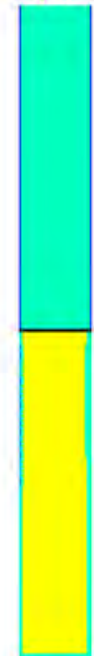

(c)

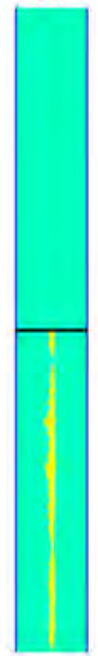

Figure 9 - Air velocity profiles for Model I (a), Model II (b), and Model III (c).

\subsection{Momentum relaxation factor}

In Figure 3, using a relaxation factor of 0.1 the simulation resulted in high residuals, stabilizing after 2.5 seconds of simulation. This behavior is common in simulations, since at the start of the numerical calculations the variables are intensively changing in short periods of time, especially in transient state systems.

By reducing the relaxation factor by ten times, the residuals were smaller and less fluctuating. Thus, the problem converged faster and in a less chaotic way. Figure 3 demonstrates that the residuals quickly lower to a value similar to the values obtained after the stabilization of the 0.1 relaxation factor simulation.

After reducing the relaxation factor by one hundred times, the behavior of the residual was smaller than the residuals for a relaxation factor of 0.1. However, the mass residual initially oscillated in peaks, and after stabilization, its values were higher than those found for the other two simulations.

The residual results imply that the reduction of the relaxation factor can be positive in assisting the stabilization of the residuals at the start of the simulation. However, this change needs to be moderate, as intense reductions can cause higher residuals at later time sections of the simulation. Thus, it was concluded that the moderate reduction of the relaxation factor can be useful for the early stages of the simulation, in which the convergence is usually more difficult. 
As seen in Figure 4, the particle movement was similar between the 0.1 and 0.01 values. Although some movement was still observed closer to the particle height, their behavior approximated to the static state expected by a fixed bed operation.

For the value of 0.001 there was a drastic change to the particle behavior. The solid quantity was reduced in comparison to the initial conditions, implying the particles were dragged out of the equipment at the beginning of the simulation, which represents an experimental inconsistency. In addition, after the stabilization of the system, an intense particle movement still persisted.

This result reiterates the importance of the moderate reduction of the relaxation factor. To excessively reduce the relaxation factor implies the addition of accentuated inertias to the momentum variables of the system. This is shown by the behavior for the particles with a factor of 0.001, in which the solid, initially stagnant, takes too much time to react to the drying air velocities. It is possible the stagnant particles with strong inertias hindered the air flow, creating air pockets responsible for dragging part of the particles out of the equipment at the start of the simulation.

In all simulations, presented in Figure 5, the air velocity was higher in sections with the presence of solid. This is coherent, as the solid reduces the flow area of the air. The results were coherent with the observed by Stoppe, Neto, and Santos (2020), in which the air velocities of a fixed bed dryer increased significantly along the axis of the equipment, with the higher velocities at the top exceeding the boundary value.

The usage of smaller relaxation factors resulted in smaller values of air velocity inside the equipment. This is consistent considering the inertial conservation effect of the relaxation factor reduction. The velocity values changed moderately between the values of 0.1 and 0.01 , and significant changes were observed for the value of 0.001 . The changes of air velocity for the 0.001 relaxation factor were specially situated closer to the particle height, due to the previously observed sweep of the particles.

\subsection{Influence of models}

As seen in Figure 6, the residual behavior for Model II showed fast and efficient convergence, stabilizing quickly with smaller residuals in comparison to Model I.

The use of Model III, however, caused a significant increase to the residuals. Although the residual oscillated in a fixed amplitude without the formation of peaks, its value was considerably higher to those observed for Model I and II.

The simulations configured with all three different models were calculated until the simulation time reached 42 seconds. The volumetric fraction, seed temperature and air velocity profiles were obtained using a 0.1 relaxation factor for Model II and III, and 0.01 for Model I.
As seen in Figure 7, the particle behavior for Model II was as expected for the packed bed configuration, with no particle movement, as stated by Souza, Miranda, and Barrozo (2015). Thus, considering particle behavior, Model II was better suited for the modelling of the fixed bed.

In Figure 8, for Model I, the heat exchange between the phases was approximately zero. To clarify this, a hypothesis was raised: there may exist incompatibility between the constant heat transfer coefficient modelling and EMMG with packed bed disabled. For simulations using fluidized or spouted beds, the heat transfer could be modelled more successfully using the usual and recommended models for EMMG, dependent of the Reynolds number of the particle. As this work studied the fixed bed system, these models are not applicable since the Reynolds number for the particle would be zero.

Comparably, a more intense heat exchange occurred for Model II, with a moderate rise in temperature for the bottom particles of the bed despite the low simulation time. This allows for the conclusion that the constant heat transfer coefficient modelling is more compatible to the EMMG with packed bed configuration enabled.

The heating for Model II was gradual throughout the equipment, resulting in a temperature gradient, such as observed by Coradi and Lemes (2019). Due to the small simulation time of 42 seconds, the heating velocity is not easily comparable, but it can be observed for this model a considerably fast heating of the beans, likely surpassing the heating velocity obtained by Coradi and Lemes (2019). This should be further studied, as a rapid heating of the beans could present itself as an inconsistency with experimental results.

For Model III the particle was also considerably heated, but to smaller extents. A hypothesis for the slower heat transfer in Model III is the effective thermal conductivity used in the porous zone model. The thermal conductivity, used in the energy balance, is the average conductivity of both phases. Thus, the heat transfer reduction might have been due to differences between the conductivity of both phases, as the air conductivity is lower than the particle conductivity.

As seen in Figure 9, in all simulations the air velocity was more intense in the solid region, which is coherent due to the flow area reduction in this section. For Model II, the velocities were considerably homogeneous, with smaller values compared to the ones obtained for Model I with a 0.01 relaxation factor. For Model III, the velocities were also homogeneous, but in lower velocities, with the exception of the central section of the equipment. The values of air velocity for Model III suggests an overestimation of the momentum drops in the equipment.

It is important to highlight how precise and easily displayable the variable profiles are. The temperature, for 
example, as a very important parameter in coffee drying, could easily be monitored over time with an average value, or various precise and local measurements along the equipment. This shows itself as one of the many advantages of using CFD for the modelling of equipment.

The same was true for the volumetric fraction, which, with CFD, it was possible to simulate the entire bed and determine the fluidization regime. An important next step would be the integration of moisture content in the simulation, but applying a model suitable for thick layer drying. Models that simulate singular beans like the one proposed by Amaral et al. (2018) are useful for a small scale, thin layer application, so adaptations must be made in order to guarantee representability.

\section{CONCLUSIONS}

Reductions to the momentum relaxation factor assisted the numerical convergence and resulted in less residuals. Intense reductions, however, altered the particle movement and air velocities, causing unwanted behaviors.

The EMMG with packed bed configuration enabled (Model II) had the lowest residuals and good convergence of the temperature and volumetric fraction, highlighting it as a good modelling alternative for convergence improvement. The EMMG with disabled packed bed configuration resulted in converging heterogeneous velocities to the literature, but the heat transfer was approximately nonexistent, and the particles at the top were not static. Therefore, it might not be recommended as a good model for this system at the velocity value used. The porous zone modelling had similar results to Model II, but with slower heat transfer, lower air velocity values, and more residuals.

In general, the numeric results for Model II were the most promising, suggesting it as a good alternative for fixed bed drying modelling. Its use can reduce the computational costs and better represent the behavior of the system. With an optimized numerical method, an opportunity opens up for the evolution of the numerical methodology with the addition of a moisture content modelling, allowing for computational predictions of seed drying in a fast and economical way.

\section{ACKNOWLEDGEMENTS}

This work was supported by the, Foundation for Research Support of Minas Gerais (FAPEMIG) project number APQ-00022-18, National Council for Scientific and Technological Development (CNPq) project number 427962/2018-8 and Federal University of Lavras (UFLA) project numbers PIDEG357-2018 and PIDEG76-2018, the authors are grateful to the for their financial support.

\section{REFERENCES}

AFONSO, P. C. et al. Shrinkage evaluation of five different varieties of coffee berries during the drying process. Biosystems Engineering, 86(4):481-485, 2003.

AMARAL, R. et al. Simulation of coffee fruit drying using computational fluid dynamics, Coffee Science, 13(4):477488, 2018.

ANSYS, Inc. ANSYS Fluent user's guide. Southpointe. Canonsburg, 2013. Available in: <http://www.pmt.usp.br/ ACADEMIC/martoran/NotasModelosGrad/ANSYS $\% 20$ Fluent\%20Users\%20Guide.pdf $>$ Access in: June, 22, 2020.

AZADBAKHT, M. et al. Thermal properties of soybean pod as a function of moisture content and temperature. American journal of food science and technology, 1(2):9-13, 2013.

BORÉM, F. M. et al. Thermal properties of five different species of peeled cofee. Revista Brasileira de Engenharia Agrícola e Ambiental, 6(3):475-480, 2002.

BORÉM, F. M. et al. Effect of intermittent drying and storage on parchment coffee quality. Bioscience Journal, 30(5):609-616, 2014.

CHIESA, M. et al. Numerical simulation of particulate flow by the eulerian-lagrangian and the eulerian-eulerian approach with application to a fluidized bed. Computers \& chemical engineering, 29(2):291-304, 2005.

COFFEE STATISTICAL REPORT. Dezembro 2019. Available at: < http://www.consorciopesquisacafe.com.br/ index.php/2016-07-18-14-42-22\#a $>$ Accessed on: June, 28, 2020. (In Portuguese)

CORADI, P. C. et al. Mathematical modeling of drying in a new concept of silo-dryer-aerator and the quality of soybean seeds (Glycine max (L.) Merrill). Journal of Agricultural Science and Technology B, 8(2):483-498, 2018.

COSTA, F. O.; PERES, A. P.; PETRI JUNIOR, I. Simulation of hydrocyclones for evaluating the flow of nonNewtonian fluids. Revista de Engenharia e Tecnologia, 13(1):1-12, 2021

CORADI, P. C.; LEMES, Â. F. C. Experimental prototype of silo-dryer-aerator of grains using Computational Fluid Dynamics (CFD) system. Acta Scientiarum. Technology, 41(1):e36949, 2019.

DEFENDI, R. O.; PARAÍSO, P. R.; JORGE, L. M. M. Optimization study of soybean intermittent drying in fixed-bed drying technology. Drying Technology, 35(1):125-137, 2017. 
GHOSH, P.; VENKATACHALAPATHY, N. Processing and drying of coffee-a review. International Journal of Engineering Research \& Technology, 3(12):784-794, 2014.

HAMZEHEI, M. CFD modeling and simulation of hydrodynamics in a fluidized bed dryer with experimental validation. International Scholarly Research Notices, 2011(1):1-9, 2011.

ISMAIL, T. M. et al. Eulerian-eulerian CFD model on fluidized bed gasifier using coffee husks as fuel. Applied Thermal Engineering, 106(1):1391-1402, 2016.

PALACIN, J. J. F. et al. Combined drying of peeled coffee cherry. Revista Engenharia na Agricultura Reveng, 17(3):244-258, 2009.

SANTOS, D. A. et al. Mixture of particles' influence in computer simulations of a spouted bed. Materials Science Forum, 660(1):488-453, 2010.

SOPONRONNARIT, S. et al. Fluidised bed drying of soybeans. Journal of Stored Products Research, 37(2):133-151, 2001.

SOUZA, G. F. M. V.; MIRANDA, R. F.; BARROZO, M. A. S. Soybean (Glycine max L. Merrill) seed drying in fixed bed: Process heterogeneity and seed quality. Drying Technology, 33(14):1779-1787, 2015.

STOPPE, A. C. R.; NETO, J. L. V.; DOS SANTOS, K. G. Development of a fixed bed solar dryer: Experimental study and CFD simulation. Research, Society and Development, 9(3):e123932667, 2020.

SZAFRAN, R. G.; KMIEC, A. C. F. D modeling of heat and mass transfer in a spouted bed dryer. Industrial \& engineering chemistry research, 43(4):1113-1124, 2004.
RESENDE, O. et al. Mathematical modelling of the drying process for two varieties of beans (Phaseolus vulgaris L.). Revista Brasileira de Produtos Agroindustriais, 10(1):17-26, 2008.

WELCH, G. B. Seed processing in Brasil. Brasília, Distrito Federal: Ministério da Agricultura, 1980. 205p.

MUJUMDAR, A. S. Handbook of industrial drying, revised and expanded. New York, NY: CRC Press, 2006. 1423p.

VERSTEEG, H. K.; MALALASEKERA, W. An introduction to computational fluid dynamics: The finite volume method. Harlow, United Kingdom: Pearson education, 2007. 503p.

VICENTE, W. et al. An Eulerian model for the simulation of an entrained flow coal gasifier. Applied Thermal Engineering, 23(15):1993-2008, 2003.

WANG, H. G. et al. Investigation of batch fluidized $\square$ bed drying by mathematical modeling, CFD simulation and ECT measurement. AIChE Journal, 54(2):427-444, 2008.

WANG, Y. et al. CFD simulations of membrane filtration zone in a submerged hollow fibre membrane bioreactor using a porous media approach. Journal of Membrane Science, 363(1):57-66, 2010.

WELTY, J.; RORRER, G. L.; FOSTER, D. G. Fundamentals of momentum, heat, and mass transfer. 6. ed. New Jersey: John Wiley \& Sons, 2014. 768p.

XIA, B.; SUN, D. W. Applications of computational fluid dynamics (CFD) in the food industry: a review. Computers and electronics in agriculture, 34(1-3)5-24, 2002. 